\title{
Transferências voluntárias e corrupção municipal no Brasil: evidências preliminares do cadastro de contas irregulares do TCU
}

\author{
Carlos Leonardo Kulnig Cinelli*
}

RESUMO - Este trabalho busca complementar a evidência empírica da literatura relacionada a transferências governamentais e corrupção municipal no Brasil. Trabalha-se com dados em painel, de 1998 a 2008, oriundos do Cadastro de Contas Irregulares do TCU, das transferências voluntárias do Tesouro Nacional, bem como outras variáveis socioeconômicas de controle. Encontram-se evidências de que transferências voluntárias do Governo Federal aos municípios, bem como anos de eleições para prefeitos, são estatisticamente significantes para se explicar corrupção, corroborando os resultados encontrados em estudos anteriores.

Palavras-chave: Corrupção. Transferências voluntárias. TCU.

\section{INTRODUÇÃO}

A corrupção governamental é, muito provavelmente, um dos maiores problemas enfrentados pelas economias em desenvolvimento. Modelos teóricos, bem como estudos empíricos, acusam-na como a fonte de efeitos deletérios diversos, impactando diretamente no crescimento e desenvolvimento econômicos, tais como: (i) a redução do investimento doméstico e externo ao país corrupto; (ii) a intensificação das desigualdades econômicas; (iii) a limitação do poder de atuação do Estado em políticas econômicas pró-crescimento; (iv) a alocação de recursos de maneira ineficiente; (v) a quebra do rule of law, de maneira a impedir o florescimento da estrutura institucional necessária à promoção dos mercados, entre outros elencados pela literatura (ACEMOGLU; VERDIER, 1998; BAI; WEI, 2000; BARDHAN, 1997; BURKY; PERRY, 1998; KAUFMAN; KRAAY; ZOIDO-LOBATÓN, 1999; KNACK; KEEFER, 1995; LI; XU; ZOU, 2000; MAURO, 1995; ROCK; BONNETT', 2004).

O afã de se estudar o assunto, entretanto, encontra obstáculos empíricos: medir a corrupção não é tarefa fácil. Assim, o intuito de verificar relações empíricas das causas ou efeitos da corrupção governamental doméstica passa por identificar proxies para a corrupção. No caso do Brasil, os dados dos relatórios de auditoria, por sorteio, da Controladoria Geral da União (CGU) têm sido a solução encontrada. Dentre os trabalhos que utilizaram esta base de dados, encontramos o de Alves e Sodré (2007), sugerindo que transferências intergovernamentais voluntárias, via emendas parlamentares, impactam positivamente no número de corrupções municipais; o de Brollo (2008), em que se verifica que municípios corruptos tendem a ser penaliza-

\footnotetext{
* Mestrando em Economia pela Universidade de Brasília. É analista da Agência Nacional de Águas. Endereço
} eletrônico: carlos.cinelli@ana.gov.br. 
dos com menos transferências discricionárias do Governo Federal; o de Ferraz e Finnan (2008), que indicam que prefeitos corruptos auditados têm menor probabilidade de serem reeleitos; e, interessante citar, o de Brollo et al. (2010), que, em trabalho mais elaborado, encontram fortes indícios de que o aumento de repasses federais aos municípios incrementa a corrupção.

Em tentativa, malograda, de construir um indicador estadual de corrupção, Boll (2010) acaba por levantar uma base de dados interessante, em painel, do Tribunal de Contas da União (TCU), cujos casos de corrupção, diferentemente dos relatórios da CGU, foram julgados irregulares pela Corte de Contas brasileira. Tais casos tratam-se, possivelmente, de fatos mais próximos da corrupção stricto sensu do que a mera menção de irregularidade encontrada nos relatórios de auditoria. Tendo em vista os resultados de Alves e Sodré (2007), bem como os de Brollo et al. (2010), o presente trabalho procura verificar por meio de proxies alternativas, com dados em painel, se é mantida a relação existente entre transferências e corrupção, buscando examinar a robustez dos resultados já encontrados nos estudos supracitados. O texto, no que segue, está composto da seguinte forma: além desta introdução, a segunda seção trata dos dados coletados e do modelo econométrico utilizado. A terceira seção apresenta os resultados obtidos e, por fim, são tecidas algumas considerações a guisa de conclusão.

\section{DADOS E MODELO ECONOMÉTRICO}

São utilizados dados em painel dos 26 estados e do Distrito Federal, referentes aos anos de 1998 a 2008. As variáveis tomadas como proxy para corrupção governamental são o número de processos julgados irregulares pelo TCU e o valor (em milhões) dos processos julgados irregulares pelo TCU, a partir da base Cadirreg. Os dados podem ser encontrados em Boll (2010). As variáveis explicativas de destaque para o modelo são os dados relativos às transferências voluntárias da União aos municípios (em milhões), fornecidos pelo Tesouro Nacional, bem como dummies relativas ao ano de eleição para prefeitos. A escolha dessas variáveis tem por base os resultados já apresentados na literatura - em especial Brollo et al. (2010) e Ferraz e Finan (2008). De modo a compatibilizar com os dados do Cadirreg, as transferências são agregadas por UF. Com relação aos anos eleitorais, a dummy de eleição para prefeitos assume o valor 1 para os anos de 2000, 2004 e 2008 e zero caso contrário. Apesar de o foco do trabalho ser a corrupção municipal, foram igualmente coletadas as transferências voluntárias da União aos estados e incluída a variável dummy de anos de eleição para governador.

Tentando aproximar-se de uma captura dos efeitos ceteris paribus de transferências voluntárias no nível de corrupção, bem como atenuar possível viés de variável omitida, algumas variáveis de controle foram selecionadas. Assim, séries socioeconômicas usuais serviram de base 
para a estimação, tais como: (i) o número de municípios do estado - como medimos o número de irregularidades, quanto mais prefeituras em um dado estado, possivelmente maiores são as chances de alguma irregularidade ser cometida; (ii) PIB real - o nível de riqueza de um estado pode impactar o nível de corrupção tanto positivamente (aumentando a oportunidade de desvios) quanto negativamente (estados mais ricos podem bancar melhor combate à corrupção); (iii) desemprego, concentração de renda (Gini), grau de informalidade do trabalho, número de pessoas em pobreza extrema - essas variáveis socioeconômicas são proxies que tentam isolar os efeitos da pobreza e desigualdade social no nível de corrupção; (iv) escolaridade dos homens e mulheres, assim como o grau de analfabetismo do estado - uma população mais educada pode controlar seus governantes, ou, por outro lado, uma elite governante mais bem educada pode ter mais facilidade de desviar recursos públicos; (v) população residente e área - o número de pessoas residentes no estado e seu tamanho também podem ter efeitos sobre o nível de corrupção, ou serem altamente correlacionadas com variáveis omitidas, e incluí-las garante-nos, no mínimo, ter o efeito das transferências voluntárias nas irregularidades, mantendo-se fixos a população residente e o tamanho do estado; (vi) dummies para os partidos que governavam o estado - diferentes partidos do governo do estado podem ter, naturalmente, níveis maiores ou menores de corrupção. Todas as variáveis foram obtidas junto ao IPEADATA.

As variáveis dependentes mostram grande dispersão entre os estados, com número médio de 21 processos irregulares, de $\mathrm{R} \$ 16$ milhões de desvios de recursos públicos, e desvios-padrão de 21 processos e $\mathrm{R} \$ 86$ milhões respectivamente. Este alto coeficiente de variação $(5,15)$ do valor dos desvios de recursos públicos chama a atenção, e é interessante verificar a presença de outliers. De fato, o valor dos desvios no estado de São Paulo no ano de 1999, que segundo os dados de Boll (2010) alcançou a cifra de R \$ 1,46 bilhão, é cerca de noventa vezes maior do que a média. Este valor é demasiado grande e pode se tratar, possivelmente, de um erro de mensuração ou de digitação quando da tabulação dos dados, e verificaremos que será importante levar isso em consideração nas regressões. A média e o desvio-padrão dos valores dos processos irregulares, sem o outlier, ficam em $\mathrm{R} \$ 12$ milhões e $\mathrm{R} \$ 18$ milhões, respectivamente. As transferências voluntárias, bem como as demais variáveis explicativas apresentam-se da mesma forma, com variância significativa. Com relação às dummies referentes aos partidos, verifica-se que o PSDB esteve presente em $24 \%$ da amostra (anos x UF) no período analisado, seguido do PMDB (21\%), PT e PFL (14\%).

Buscando avaliar se a as transferências voluntárias aos municípios impactam nos números e valores dos processos irregulares, agrupados por estado, os modelos básicos deste trabalho serão: 


$$
\begin{aligned}
\text { nirreg }_{i t}= & \beta_{1} \text { transfm }_{i t}+\beta_{2} \text { transfm }_{i t}^{2}+\beta_{3} \text { eleiçãoprefeito }_{i t}+\beta_{4} \text { tranfe }_{i t}+ \\
& +\beta_{5} \text { transfe }_{i t}^{2}+\beta_{6} \text { eleiçãogovernador }_{i t}+X \gamma+\varepsilon_{i t} \\
\text { virreg }_{i t}= & \beta_{1} \text { transfm }_{i t}+\beta_{2} \text { transfm }_{i t}^{2}+\beta_{3} \text { eleiçãoprefeito }_{i t}+\beta_{4} \text { tranfe }_{i t}+ \\
& +\beta_{5} \text { transfe }_{i t}^{2}+\beta_{6} \text { eleiçãogovernador }_{i t}+X \gamma+\varepsilon_{i t}
\end{aligned}
$$

onde nirreg representa o número de processos julgados irregulares no TCU, virreg o valor dos processos julgados irregulares no TCU, transfm o valor das transferências voluntárias aos municípios dos estados, transfe o valor das transferências voluntárias aos governos dos estados, eleiçãoprefeito a dummy indicando anos de eleições para prefeito e eleiçãogovernador a dummy indicando anos de eleição para governador. Por fim, $X$ é o vetor das variáveis de controle (e $\gamma$ o vetor de coeficientes correspondentes), abarcando uma constante. Incluiu-se o termo quadrático para se permitir uma relação não linear (tanto $\operatorname{com} \beta_{2}, \beta_{5}>0$ quanto $\operatorname{com} \beta_{2}, \beta_{5}<0$ ) entre corrupção e transferências voluntárias. Espera-se para $\beta_{1}$ e $\beta_{4}$ sinal positivo e de magnitude com relevância econômica.

Para o presente trabalho, um problema relevante que se enfrenta ao se realizarem as regressões é o viés de variável omitida. Para tanto, uma possibilidade seria incluir as variáveis de controle já mencionadas. Contudo, isto pode não ser suficiente. Assim, além das variáveis de controle trabalharemos com modelos de efeito fixo, que eliminem prováveis variáveis omitidas que sejam constantes ao longo do tempo, atenuando este problema.

\section{RESULTADOS}

A Tabela 1 apresenta os resultados das regressões tendo como variável dependente o número de irregularidades no Cadirreg por UF. Primeiramente, fez-se uma regressão por mínimos quadrados agrupados, utilizando como variáveis independentes apenas as transferências voluntárias e anos eleitorais. Os resultados apresentam-se de acordo com o esperado pela teoria, com um coeficiente de 0,14 para as transferências voluntárias aos municípios - cabe frisar que esta magnitude é alta, grosso modo estima que, na média, para cada $\mathrm{R} \$ 7$ milhões a mais de transferências voluntárias aos municípios, há mais um caso de corrupção registrado.

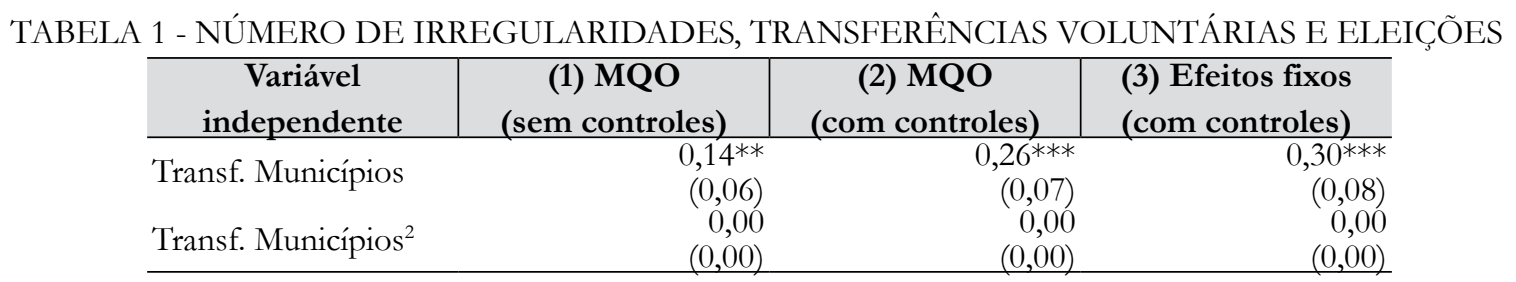


TABELA 1 (CONTINUAÇÃO) - NÚMERO DE IRREGULARIDADES, TRANSFERÊNCIAS VOLUNTÁRIAS E ELEIÇÕES

\begin{tabular}{lrrr|r}
\hline \multicolumn{1}{c|}{$\begin{array}{c}\text { Variável } \\
\text { independente }\end{array}$} & $\begin{array}{c}\text { (1) MQO } \\
\text { (sem controles) }\end{array}$ & $\begin{array}{c}\text { (2) MQO } \\
\text { (com controles) }\end{array}$ & $\begin{array}{c}\text { (3) Efeitos fixos } \\
\text { (com controles) }\end{array}$ \\
\hline Eleições Prefeitos & 1,27 & $9,34^{* * *}$ & $9,77^{* * *}$ \\
& $(1,42)$ & $(3,04)$ & $(3,31)$ \\
Transf. Estados & 0,07 & $-0,05$ & $-0,05$ \\
& $(0,05)$ & $(0,04)$ & $(0,05)$ \\
Transf. Estados ${ }^{2}$ & 0,00 & 0,00 & 0,00 \\
& $(0,00)$ & $(0,00)$ & $(0,00)$ \\
Eleições Governador & $3,53 *$ & $-0,44$ & $-3,21^{*}$ \\
Observações & $(2,04)$ & $(2,21)$ & $(1,94)$ \\
R2 ajustado & 297 & 216 & 216 \\
\hline
\end{tabular}

FONTE: Cálculo do autor.

NOTA: Variável dependente: número de processos irregulares no Cadirreg por UF; erros-padrão robustos em parênteses; * para significância estatística a $10 \%$, ** para significância estatística a $5 \%$ e *** para significância estatística a $1 \%$; as estimativas dos coeficientes das variáveis de controle e da constante foram omitidas da tabela; Teste de Hausman, $p$-valor $=0,01$ (retiradas as covariadas que não se mostraram conjuntamente significantes para possibilitar a regressão de efeitos aleatórios); apesar de os valores para os termos quadráticos serem zero quando arredondados para duas casas decimais, rejeita-se (teste F) que ambos sejam iguais a zero; as transferências encontram-se em milhões.

Entretanto, na estimação anterior há grandes chances de se ter um viés de variável omitida. Por exemplo, suponha que o número de pessoas na extrema pobreza seja correlacionado com as transferências municipais voluntárias, uma vez que o Governo Federal deseje acabar com a miséria; ademais, por outro lado, suponha que esta variável também seja correlacionada com os casos de corrupção, pois uma população com mais miseráveis teria menos condições de vigiar seus governantes. Neste caso, a omissão do número de pessoas em extrema pobreza nos daria estimadores inconsistentes. Raciocínio análogo pode ser feito com todas as demais variáveis de controle. Assim, a segunda coluna da Tabela 1 evidencia a regressão por mínimos quadrados agrupados incluindo-se todos os controles socioeconômicos. Uma desvantagem da inclusão dos controles é que passamos de 297 observações para 216, pois para alguns anos não temos dados. Contudo, espera-se que isto não cause problemas nas estimativas, uma vez que a fonte da falta de dados é exógena (anos de censo, por exemplo) e não está correlacionada com nenhuma das variáveis em questão. O resultado desta regressão nos dá um coeficiente para transferências voluntárias aos municípios quase duas vezes maior do que na regressão anterior - na média, para cada $\mathrm{R} \$ 3,8$ milhões a mais de repasse do Governo Federal, teríamos um caso extra de corrupção registrado. Além disso, a dummy de eleições para prefeito mostra-se bastante significativa - em anos eleitorais teríamos, em média, cerca de nove casos de corrupção a mais do que nos demais anos.

Obviamente, apesar da extensa lista de controles, difícil imaginar que incluímos todos aqueles necessários. Decerto, há alguns fatores institucionais, intrínsecos a cada estado - como, 
talvez, a presença do notório poder da família Sarney no Maranhão - que explicam diferenças transversais no nível de corrupção, e que estão correlacionados com o nível de transferências do Governo Federal, mas que, felizmente, não variam no período de tempo analisado. Dessa forma, apesar de não possuirmos estes dados, podemos controlar a presença dessas variáveis omitidas utilizando uma estimação por efeitos fixos. A última coluna da Tabela 1 apresenta esta regressão, que fortalece os resultados obtidos na coluna anterior, fornecendo-nos um coeficiente de 0,30 para transferências voluntárias e de 9,77 para anos de eleições de prefeitos.

Passemos agora às regressões, encontradas na Tabela 2 , cuja variável dependente é o montante desviado nos casos de corrupção julgados pelo TCU. A primeira coluna da tabela apresenta a regressão por mínimos quadrados agrupados e os resultados são bastante conflitantes com os verificados na Tabela 1 , pois as transferências voluntárias aos municípios têm coeficiente nulo e as dummies de anos eleitorais têm coeficientes negativos. Contudo, como mencionado anteriormente, há grande potencial de viés de variável omitida, e a segunda coluna nos mostra os resultados de mínimos quadrados agrupados incluindo-se as variáveis de controle. Não obstante, novamente os resultados não corroboram aqueles da Tabela 1, tendo o coeficiente de transferências aos municípios, inclusive, sido estimado com valor negativo.

TABELA 2 - VALOR DAS IRREGULARIDADES, TRANSFERÊNCIAS VOLUNTÁRIAS E ELEIÇÕES

\begin{tabular}{|c|c|c|c|c|}
\hline $\begin{array}{c}\text { Variável } \\
\text { independente }\end{array}$ & $\begin{array}{c}\text { (1) MQO } \\
\text { (sem controles) }\end{array}$ & $\begin{array}{c}\text { (2) MQO } \\
\text { (com controles) }\end{array}$ & $\begin{array}{l}\text { (3) Efeitos Fixos } \\
\text { (com controles) }\end{array}$ & $\begin{array}{c}\text { (4) Efeitos fixos } \\
\text { (controles e outlier } \\
\text { dumm } \gamma)\end{array}$ \\
\hline \multirow{2}{*}{ Transf. Municípios } & 0,00 & $-0,51$ & $0,84 * * *$ & $0,11^{*}$ \\
\hline & $(0,07)$ & $(0,49)$ & $(0,27)$ & $(0,06)$ \\
\hline \multirow{2}{*}{ Transf. Municípios ${ }^{2}$} & 0,00 & 0,00 & 0,00 & 0,00 \\
\hline & $(0,00)$ & $(0,00)$ & $(0,00)$ & $(0,00)$ \\
\hline \multirow{2}{*}{ Eleições Prefeitos } & $-11,07$ & $-0,33$ & $-0,80$ & $5,41 * *$ \\
\hline & $(10,36)$ & $(4,15)$ & $(6,54)$ & $(2,33)$ \\
\hline \multirow{2}{*}{ Transf. Estados } & 0,22 & 0,45 & $0,74^{*}$ & $-0,06$ \\
\hline & $(0,31)$ & $(0,45)$ & $(0,42)$ & $(0,04)$ \\
\hline \multirow[t]{2}{*}{ Transf. Estados ${ }^{2}$} & 0,00 & 0,00 & 0,00 & 0,00 \\
\hline & $(0,00)$ & $(0,00)$ & $(0,00)$ & $(0,00)$ \\
\hline Eleições Governador & $\begin{array}{r}-9,06 \\
(11,80) \\
\end{array}$ & $\begin{array}{r}6,54 \\
(4,28) \\
\end{array}$ & $\begin{array}{r}-15,94 * \\
(8,32)\end{array}$ & $\begin{array}{r}0,24 \\
(2,86)\end{array}$ \\
\hline Observações & 297 & 216 & 216 & 216 \\
\hline R2 ajustado & 0,01 & 0,13 & 0,34 & 0,98 \\
\hline
\end{tabular}

FONTE: Cálculo do autor.

NOTA: Variável dependente: valor (em milhões) das irregularidades no Cadirreg por UF; erros-padrão robustos em parênteses; $*$ para significância estatística a $10 \%$, ** para significância estatística a $5 \%$ e *** para significância estatística a $1 \%$; as estimativas dos coeficientes das variáveis de controle e da constante foram omitidas da tabela; Teste de Hausman, $p$-valor $=0,00$ (retiradas as covariadas que não se mostraram conjuntamente significantes para possibilitar a regressão de efeitos aleatórios); apesar de os valores para os termos quadráticos serem zero quando arredondados para duas casas decimais, rejeita-se (teste F) que ambos sejam iguais a zero; a outlier dummy refere-se à atribuição do valor 1 a São Paulo no ano de 1999, cujo valor no Cadirreg (BOLL, 2010) foi de R \$ 1,46 bilhão, cerca de 90 vezes maior do que a média. As tranferências encontram-se em milhões.

Os resultados das colunas 1 e 2 da Tabela 2, quando comparados com a Tabela 1, 
sugerem, assim, que a presença de efeitos fixos não observados, para os valores dos casos de corrupção, podem ter impacto bem mais significativo do que para o número de casos de corrupção. Dessa forma, a terceira coluna apresenta as estimativas dos coeficientes utilizando-se o modelo de efeitos fixos. De fato, de estimativas estatisticamente insignificantes, passamos para estimativas de magnitudes bastante altas, de 0,84 e 0,74 para os coeficientes de transferências voluntárias aos municípios e aos estados, respectivamente. Tal modelo nos indicaria que, em média, a cada $\mathrm{R} \$ 1$ milhão transferido, teríamos $\mathrm{R} \$ 840$ mil desviados, se fosse para as prefeituras, e R $\$ 740$ mil no caso dos governos estaduais. Trata-se, evidentemente, de uma estimativa bastante implausível, muito provavelmente em decorrência do outlier já identificado na seção anterior.

Tendo isso em vista, a quarta coluna da Tabela 2 mostra os resultados da regressão considerando-se uma dummy para o outlier, isto é, uma dummy para São Paulo no ano de 1999. Percebe-se que a mera inclusão desta variável faz com que o $\mathrm{R}$ quadrado ajustado salte de 0,34 para 0,98 , ilustrando o peso desta observação na variabilidade dos dados e, por conseguinte, nas estimativas. Os resultados dessa regressão contam uma história bem similar às evidências encontradas na Tabela 1 - agora, a cada R \$ 1 milhão de transferências voluntárias aos municípios, tem-se que, em média, cerca de R $\$ 110$ mil são desviados. Além disso, anos de eleição para prefeitos apresentam, na média, cerca de $\mathrm{R} \$ 5,4$ milhões a mais de recursos desviados do que nos demais anos. Antes de passarmos às considerações finais, cabe mencionar que foram realizados testes de Hausman tanto na regressão (3) da Tabela 1 quanto na regressão (4) da Tabela 2 rejeitando-se, em ambas, a hipótese nula de que as estimativas por efeitos aleatórios são consistentes. Além disso, também foram feitas essas mesmas regressões por primeiras diferenças, obtendo-se resultados similares e, portanto, essas foram omitidas.

\section{CONSIDERAÇÕES FINAIS}

Nesse trabalho, buscamos complementar a evidência empírica da literatura, analisando a relação entre transferências voluntárias e nível de corrupção municipal, por meio de base de dados alternativa, proveniente do TCU. Verificou-se que se manteve o fato estilizado de que maiores transferências aos municípios acompanham, na média, uma maior quantidade de irregularidades: cada $\mathrm{R} \$ 3,3$ milhões a mais transferidos aos municípios tendem a gerar, na média, acréscimo de 1 processo irregular no Cadirreg, bem como $\mathrm{R} \$ 363$ mil desviados. Outro fato que se mostrou consistente ao longo dos modelos foi o de que anos eleitorais para prefeitos têm, em média, 9,77 processos irregulares a mais, bem como o acréscimo $\mathrm{R} \$ 5,41$ milhões aos desvios, do que nos demais anos. Uma análise mais detalhada e qualitativa dos processos do 
TCU complementaria os resultados mais gerais e quantitativos obtidos. Tal empreitada, contudo, foge ao escopo do presente trabalho. Juntando-se esses resultados aos dos demais estudos citados ao longo do texto, resta cada vez mais evidente a problemática da corrupção municipal no Brasil. Identificando o Governo Federal a necessidade de se transferirem mais recursos aos municípios, para melhorias no provimento de bens públicos, a iniciativa terá de passar por aumento significativo das irregularidades - o trade-off sugerido por Acemoglu e Verdier (2000) mostra-se, a princípio, robusto. Estudos quantitativos como os apresentados dão alguns indícios do tamanho do problema. Entretanto, necessários se fazem estudos com a desagregação por município, utilizando técnicas de estimação tobit em painel, por exemplo. Além disso, em um país federalista, não basta trabalhar apenas a corrupção com os recursos do Governo Federal: buscar meios de mensurar e analisar a corrupção de cada esfera da União, com seus recursos próprios, é frente de pesquisa teórica e empírica promissora para fornecer subsídios mais concretos para o combate à corrupção.

\section{REFERÊNCIAS}

ACEMOGLU, D.; VERDIER, T. Property rights, corruption and the allocation of talent: a general equilibrium approach. Economic Journal, v. 108, n. 450, p. 1381-1403, 1998.

ACEMOGLU, D.; VERDIER, T. The choice between market failure and corruption. American Economic Review, v. 90, n. 1, p. 194-211, 2000.

ALVES, M.; SODRÉ, A. Transferências intergovernamentais voluntárias: relação entre emendas parlamentares e corrupção municipal no Brasil. In: CONCURSO DE MONOGRAFIAS DA CGU, 2., 2007. Brasília.

BAI, C.; WEI, S. Quality of bureaucracy and open-economy macro policies. NBER Working Paper, n. 7766, 2000.

BARDHAN, P. Corruption and development: a review of issues. Journal of Economic Literature, v. 35, n. 3, p. 1320-1346, 1997.

BOLL, J. A corrupção governamental no Brasil: construção de indicadores e análise da sua incidência relativa nos estados brasileiros. Dissertação (Mestrado em Economia) Pontifícia Universidade Católica do Rio Grande do Sul, Porto Alegre, 2010.

BROLLO, F. Who is punishing corrupt politicians: voters or the central government? Evidence from the brazilian anti-corruption program. IGIER Working Paper, n. 336, 2008.

BROLLO, F.; NANNICINI, T.; PEROTTI, R.; TABELLINI, G. The political resource curse. NBER Working Paper, n. 15705, 2010.

BURKY, S.; PERRY, G. Beyond the Washington Consensus: institutions matters. Washington: World Bank, 1998.

FERRAZ, C.; FINAN, F. Exposing corrupt politicians: the effects of Brazil's publicly released audits on electoral outcomes. Quarterly Journal of Economics, v. 123, n. 2, p. 703-745, 2008. 
KAUFMANN, D.; KRAAY, A.; ZOIDO-LOBATÓN, P. Aggregating governance indicators. World Bank Policy Research Working Paper, n. 2195, 1999.

KNACK, S.; KEEFER, P. Institutions and economic performance: cross-country tests using alternative institutional measures. Economics and Politics, v. 7, n. 3, p. 207-227, 1995.

LI, H.; XU, L.; ZOU, H. Corruption, income distribution, and growth. Economics and Politics, v. 12, n. 2, p. 155-182, 2000.

MAURO, P. Corruption and growth. The Quarterly Journal of Economics, v. 110, n. 3, p. 681-712, 1995.

ROCK, M.; BONNETT, H. The comparative politics of corruption: accounting for the East Asian paradox in empirical studies of corruption, growth and investment. World Development, v. 32, n. 6, p. 999-1017, 2004. 
